\title{
DIMENSIONAMENTO DO COMPLEXO AGROINDUSTRIAL DOS ESTADOS BRASILEIROS: GERAÇÃO DE RENDA, EMPREGOS E IMPOSTOS
}

\author{
Umberto Antonio Sesso Filho' \\ Lucas Trindade Borges ${ }^{2}$ \\ Patrícia Pompermayer Sesso ${ }^{3}$ \\ Irene Domenes Zapparoli4 \\ Paulo Rogério Alves Brene 5
}

Resumo: $O$ objetivo do estudo foi dimensionar o complexo agroindustrial das unidades da federação considerando o Produto Interno Bruto, empregos e impostos gerados em quatro agregados (insumos, agropecuária, indústria e serviços). A análise foi feita a partir das matrizes de insumo-produto estaduais do ano de 2008. Os resultados mostraram que os estados do Mato Grosso, Mato Grosso do Sul, Goiás e Rondônia possuíam maior importância do Agronegócio com no mínimo 40\% do Produto Interno Bruto estadual, $50 \%$ dos empregos e $41 \%$ dos impostos indiretos líquidos. A produtividade do trabalho no Agronegócio dos estados do Norte e Nordeste é aproximadamente metade que no Sudeste/Sul do Brasil, o que mostra potencial de investimentos em tecnologia, capital e qualificação profissional. A indústria sofre a maior parte da tributação variando entre $9 \%$ e $33 \%$ e poderia beneficiar-se de desoneração.

Palavras-chave: Agronegócio. Complexo agroindustrial. Insumo-produto. Empregos. Produto Interno Bruto.

\section{SIZING OF THE AGRO-INDUSTRIAL COMPLEX OF THE BRAZILIAN STATES: INCOME GENERATION, EMPLOYMENT AND TAXES}

Abstract: The objective of the study was to size the agroindustrial complex of the federation units considering Gross Domestic Product, jobs and taxes generated in four aggregates (inputs, agriculture, industry, and services). The results showed that the states of Mato Grosso, Mato Grosso do Sul, Goiás and Rondônia had greater importance of Agribusiness with at least $40 \%$ of the Gross Domestic Product $50 \%$ of jobs and $41 \%$ of net indirect taxes. The productivity of labor in the Agribusiness of the North and Northeast states is approximately half that in the Southeast / South of Brazil, which shows potential investments in technology, capital and professional qualification. The industry suffers most of the taxation ranging from $9 \%$ to $33 \%$ and could benefit from relief.

Keywords: Agribusiness. Agroindustrial complex. Input-output. Jobs. Gross Domestic Product.

\footnotetext{
1 Universidade Estadual de Londrina, Departamento de Economia, Londrina, Brasil, umasesso@uel.br, https://orcid.org/0000-0003-4691-7343

2 Universidade Estadual de Londrina, Departamento de Economia, Londrina, Brasil, borgesbr89@gmail.com, https://orcid.org/0000-0002-2931-1684

${ }_{3}^{3}$ Universidade Estadual de Londrina, Departamento de Economia, Londrina, Brasil, papomper2004@yahoo.com.br, https://orcid.org/0000-0001-5451-5470

${ }^{4}$ Universidade Estadual de Londrina, Departamento de Economia, Londrina, Brasil, zapparoli@uel.br, https://orcid.org/0000-0002-4272-4571

5 Universidade Estadual do Norte do Paraná, Departamento de Economia, Cornélio Procópio, Brasil, paulobrene@uenp.edu.br, https://orcid.org/0000-0002-7096-8282
} 


\section{DIMENSIONAMIENTO DEL COMPLEJO AGROINDUSTRIAL DE LOS ESTADOS BRASILEÑOS: GENERACIÓN DE INGRESOS, EMPLEOS E IMPUESTOS}

Resumen: El objetivo del estudio fue dimensionar el complejo agroindustrial de las unidades de la federación considerando el Producto Interno Bruto, empleos e impuestos generados en cuatro agregados (insumos, agropecuaria, industria y servicios). El análisis fue realizado a partir de las matrices de insumo-producto estatales del año 2008. Los resultados mostraron que los estados de Mato Grosso, Mato Grosso do Sul, Goiás y Rondônia poseían mayor importancia del Agronegocio con al menos el $40 \%$ del Producto Interno Bruto, el 50\% de los empleos y el $41 \%$ de los impuestos indirectos netos. La productividad del trabajo en el Agronegocio de los estados del Norte y Nordeste es aproximadamente la mitad que en el Sudeste / Sur de Brasil, lo que muestra potencial de inversiones en tecnología, capital y calificación profesional. La industria sufre la mayor parte de la tributación que varía entre el $9 \%$ y el $33 \%$ y podría beneficiarse de la deshonación.

Palabras clave: Agronegocio. Complejo agroindustrial. Insumo-producto. Empleos. Producto Interno Bruto.

\section{Introdução}

Davis e Goldberg (1957) propuseram um novo conceito de agronegócio para compreender a cadeia produtiva dos agricultores aos consumidores. A conclusão é que a cadeia do agronegócio representa a soma de todas as operações no campo, além da produção e distribuição de todos os suprimentos agrícolas mais as operações realizadas em conexão com o manuseio, armazenamento, processamento e distribuição de produtos agropecuários. Portanto, o agronegócio refere-se ao somatório de todas as operações envolvidas na produção e distribuição de alimentos e fibras, composto por quatro setores: Insumos, Agropecuária, Agroindústria e Serviços. Ainda de acordo com os autores, o agronegócio é um complexo de sistemas que tem como característica a diminuição do controle da produção pelo agricultor, onde as atividades que cabem a este se resume ao momento da produção, dependendo de terceiros para ter acesso a insumos, máquinas e equipamentos e para a comercialização e a transformação da produção.

O Brasil possui aproximadamente um quarto das terras agricultáveis do mundo e a elevada tecnologia utilizada no campo faz o agronegócio brasileiro ser um setor moderno, eficiente e competitivo no cenário internacional. Há vários fatores que contribuem para que o país aumente sua produção agrícola no longo prazo, pode-se destacar a existência de áreas inexploradas ou exploradas de forma ineficiente que, havendo investimento em tecnologia e meios de transporte para escoamento das safras, poderão ser unificadas à produção agrícola. Os estados brasileiros possuem estruturas produtivas distintas e o agronegócio está presente 
em todos, mas cada elo do complexo agroindustrial tem impactos diferentes sobre a economia regional. Esta é a motivação do estudo, pois o dimensionamento do agronegócio das unidades da federação proporcionará diferentes enfoques para aprimoramento das cadeias produtivas regionais. A pesquisa possibilitará a estimativa da importância do agronegócio para cada região em análise, a capacidade de geração de empregos e impostos para cada agregado.

Desta forma o objetivo geral deste artigo é dimensionar o agronegócio para as unidades da federação considerando o Produto Interno Bruto (PIB), empregos e impostos gerados no ano de 2008. Para tanto a cadeia produtiva do agronegócio é dividida em quatro partes, a saber: (1) Setores de insumos; (2) Agropecuária; (3) Indústria; e (4) Comércio e serviços. Por fim, destaca-se que existe uma lacuna nas pesquisas sobre o dimensionamento do agronegócio para os estados brasileiros a partir do ano de 2008, assim a proposta é dimensionar a importância do agronegócio no tocante ao PIB, emprego e impostos, a produtividade do trabalho no setor, o potencial de investimentos em tecnologia, capital e qualificação profissional na área rural e o papel da industrialização e de serviços adicionados aos produtos.

Para atingir o objetivo o texto está dividido em cinco seções incluindo esta introdução. A segunda seção expõe os estudos recentes do dimensionamento do agronegócio dos estados do Brasil a partir do uso da matriz de insumo-produto, a exemplo dos trabalhos de Finamore e Montoya (2003) e Porsse (2003) que dimensionaram o agronegócio no Rio Grande do Sul e Nunes e Parré (2013) para o estado do Paraná entre outros. Na seção seguinte apresenta a metodologia que tem por base Furtuoso e Guilhoto (2003) e Furtuoso (1998) e a fonte dos dados provenientes do Núcleo de Economia Regional da Universidade de São Paulo (NEREUS, 2017). Os resultados e discussão fazem parte da quarta seção, que trazem os valores do Produto Interno Bruto, empregos e impostos do Agronegócio dividido em quatro agregados e a quinta seção apresenta as principais conclusões.

\section{Estudos recentes sobre o Produto Interno Bruto do Agronegócio dos estados do Brasil}

Vários trabalhos foram dedicados ao estudo individualizado do PIB do agronegócio nos estados. Para os estados da Região Nordeste, pode-se destacar os trabalhos de Araújo Neto e Costa (2005) e Guilhoto et al. (2007). Araújo Neto e Costa (2005) realizaram um cálculo do PIB (Produto Interno Bruto do Agronegócio) para a formulação e direcionamento de políticas em Pernambuco. $O$ trabalho 
conceituou e caracterizou o setor do complexo agroindustrial (CAI). Na análise dos autores foram apresentadas a classificação setorial do Agronegócio pernambucano e a mensuração do PIB, sendo que os principais resultados indicaram que o PIB do agronegócio de Pernambuco representava $21,2 \%$ do PIB total do estado. Por sua vez, Guilhoto et al. (2007) analisaram a trajetória do Produto Interno Bruto do agronegócio do Brasil e do Estado da Bahia, entre 1990 e 2005 e o detalhamento em segmentos e sub-complexos de forma a estudar as participações e definir a teia de relações e fluxos econômicos que se formam devido ao agronegócio. Os resultados apresentados pelos autores apontaram que, em média, 27,7\% do PIB nacional e $25,4 \%$ do PIB baiano são compostos pelo agronegócio. Além disso, este trabalho mostrou a predominância da lavoura em relação à pecuária e a dinâmica dos segmentos do agronegócio.

A economia mineira também foi analisada pelo trabalho de Cruz et al. (2009), os quais avaliaram as transformações na estrutura produtiva do agronegócio de Minas Gerais, segundo as definições de agregado I (fornecedor de bens e insumos para a agropecuária), agregado II (agropecuária), agregado III (processamento e industrialização agrícolas) e agregado IV (distribuição agrícola). Para isso, os autores utilizaram a matriz de insumo-produto de Minas Gerais, de 1999. O agronegócio gerou rendas equivalentes a 29,76\% do PIB de Minas Gerais e 9,6\% do PIB do agronegócio nacional. Do PIB do agronegócio mineiro, os setores fornecedores de insumos para a agropecuária participaram com 20,73\%; a agropecuária, com 27,53\%; e as atividades de processamento, industrialização e distribuição, com 51,75\%. A economia mineira apresentou características de economia alimentar industrializada, considerando a participação da produção agropecuária próxima de um terço do valor total do agronegócio.

Ainda no tocante a Minas Gerais pode-se destacar o trabalho do CEPEA (2017), que também analisa o estado de São Paulo. O CEPEA (2017) é um instituto de pesquisa dentro da Escola Superior de Agricultura "Luiz de Queiroz" (Universidade de São Paulo) que estima sistematicamente o Produto Interno Bruto do Agronegócio brasileiro e dos estados de São Paulo e Minas Gerais, como mencionado, além de diversos produtos da cadeia agroindustrial. No tocante ao estado de São Paulo, o PIB do Agronegócio representa cerca de $20 \%$ do nacional. Ainda em relação à economia paulista, a participação do agronegócio é de aproximadamente $15 \%$ do PIB, gerando também perto de 15\% dos empregos formais do estado no ano de 2017. A maior parte desses empregos está na 
agroindústria (35\%) e em serviços (47\%), ficando o segmento primário com 16\%. Esses números não incluem o trabalho da mão de obra familiar, importante nas propriedades rurais menores. A participação estimada do agronegócio mineiro no PIB é de 13,80\% em 2017.

O estudo de Guilhoto et al. (2011) foi dedicado a definir e quantificar as cadeias produtivas de base familiar abordando o nível nacional, estadual e o Distrito Federal. As principais conclusões do estudo são de que o peso da participação das cadeias produtivas da agricultura de base familiar para a geração de riqueza no país, que chega a 10\% PIB nacional entre 1995 a 2005 e aproximadamente um terço do total das cadeias produtivas agropecuárias no mesmo período. Revelou-se uma maior desconcentração na participação regional do PIB da Agricultura Familiar em relação à estrutura do PIB nacional e do PIB das cadeias produtivas agropecuárias. A região Sudeste, por exemplo, representa 55\% do PIB nacional e 24\% do PIB da Agricultura Familiar. A distribuição regional do PIB do agronegócio apresenta diferenças importantes: o segmento familiar concentra-se no Sul do país, sendo também importante no Nordeste e no Sudeste. Em 2004, o PIB do agronegócio familiar nacional atingiu a cifra de $\mathrm{R} \$ 181$ bilhões, dos quais cerca de $44 \%$ ( $R \$ 80$ bilhões) e estavam concentrados na região Sul e, destes, metade estava no Rio Grande do Sul, totalizando $R \$ 40$ bilhões (R\$ de 2005). $O$ desempenho recente da agropecuária familiar e do agronegócio a ela articulada vem sendo bastante positivo com suas taxas de crescimento igualando-se às do segmento patronal.

Para a Região Sul cita-se o estudo de Sesso Filho et al. (2011). Os autores estimaram o Produto Interno Bruto (PIB) do Agronegócio da Região Sul em $\mathrm{R} \$ 125$ bilhões no ano de 2004, o que correspondia a 27\% do total do PIB do agronegócio brasileiro (460 bilhões de reais) e 39\% do PIB total regional ( $R \$ 322$ bilhões). Ainda de acordo com o estudo, o estado com maior participação no agronegócio da região foi o Rio Grande do Sul, seguido do Paraná e Santa Catarina, sendo que a geração de empregos do agronegócio correspondeu a um terço da força de trabalho da Região Sul. Para Sesso Filho et al. (2011), em termos de geração de impostos no Agronegócio a Região Sul arrecadou em 2004 o que correspondeu a 24\% do total. De forma específica, Nunes e Parré (2013) utilizaram a matriz insumo-produto estimada para o Estado do Paraná para o ano de 2007 para dimensionar o Agronegócio paranaense. Os principais resultados deste trabalho mostram que a participação do agronegócio no PIB paranaense era de cerca $30 \%$, sendo 
visualizada a redução da participação deste segmento na economia paranaense. 0 agregado III (indústria) possui forte participação no agronegócio paranaense e foi observada diversificação na estrutura produtiva responsável pelo decréscimo da relação Agronegócios/PIB. A estrutura do Estado do Paraná no ano de 2007 pode ser considerada uma economia alimentar industrializada.

Finamore e Montoya (2003) dimensionaram o agronegócio gaúcho utilizando a ferramenta insumo-produto, estes autores verificaram que para o ano de 1998 o agronegócio respondeu a preço básico e a preço de mercado por 36,27\% e 36,67\% do PIB do Estado respectivamente. Em termos relativos, verificou-se que os impostos indiretos que recaem sobre a agroindústria $(29,31 \%)$ são maiores do que a média estadual (9,74\%). O agronegócio do estado empregava em 1998 aproximadamente $47,68 \%$ do total de trabalhadores. Além disso, os resultados mostraram que o agronegócio gaúcho contribui com divisas via exportação e que existe possibilidade para implementação de programas de substituição de importações no Estado. Na mesma linha, Porsse (2003) descreveu em detalhes o cálculo do Produto Interno Bruto do Agronegócio do Rio Grande do Sul e concluiu que os resultados explicitaram que as atividades do agronegócio do absorvem quase um terço do PIB estadual e apresentam uma contribuição acima da média para a arrecadação de impostos comparativamente aos demais setores da estrutura econômica do estado.

A análise dos estudos recentes dentro do tema dimensionamento do agronegócio mostrou que foram realizadas pesquisas para alguns dos estados do Brasil como Minas Gerais, São Paulo, Pernambuco, Bahia, Rio de Janeiro, Paraná, Santa Catarina e Rio Grande do Sul. Estudos considerando os produtos do agronegócio também foram realizados. No entanto, a maior parte dos estados não possui cálculo do PIB do Agronegócio a partir do ano de 2008 e a proposta da pesquisa é preencher a lacuna calculando o Produto Interno Bruto, emprego e impostos indiretos líquidos dos agregados do Agronegócio para todas as unidades da federação.

\section{Metodologia}

Fonte dos dados

As bases de dados a serem utilizadas são fornecidas pelo Núcleo de Economia Regional da Universidade de São Paulo (NEREUS, 2017). A fonte fornece 
dados sobre o Brasil entre 1995 e 2013 e estados para o ano de 2008. A estimativa destas matrizes se baseou em Guilhoto e Sesso Filho (2005), Guilhoto e Sesso Filho (2010) e Guilhoto et al. (2010). As matrizes de insumo-produto estaduais e do Distrito Federal possuem vinte e seis setores, os cinco pertencentes ao Agronegócio são Agricultura, Pecuária e Indústrias processadoras de Alimentos, bebidas e fumo; Têxtil, vestuário e calçados e Madeira, papel e impressão.

\section{A matriz de insumo-produto e dimensionamento do agronegócio}

A metodologia para o cálculo do PIB do agronegócio baseia-se nos trabalhos de Furtuoso e Guilhoto (2003) e Furtuoso (1998), fundamentando-se na intensidade da interligação para trás e para frente da agropecuária propriamente dita. O PIB do agronegócio resulta da soma de quatro agregados: insumos, agropecuária, indústria e distribuição. O método considera, além da agropecuária propriamente dita, as atividades que alimentam e são alimentadas pela produção rural considerando a interdependência existente entre as atividades de produção. No cálculo do PIB do Agregado I (Insumos para a Agricultura e Pecuária) são utilizadas as informações referentes aos valores dos insumos adquiridos pela Agricultura e Pecuária e que estão disponíveis nas tabelas de insumo-produto, estimadas de acordo com a metodologia apresentada em Guilhoto e Sesso Filho (2005). As colunas com os valores dos insumos são multiplicadas pelos respectivos coeficientes de valor adicionado $\left(C V A_{i}\right)$, onde temos $i=26$ setores. Para obter-se os Coeficientes do Valor Adicionado por setor $\left(C V A_{i}\right)$ divide-se o Valor Adicionado a Preços de Mercado ${ }^{6}\left(V A_{P M_{i}}\right)$ pela Produção do Setor $\left(X_{i}\right)$, ou seja,

$$
C V A_{i}=\frac{V A_{P M_{i}}}{X_{i}}
$$

Desta forma, o problema de dupla contagem, comumente apresentado em estimativas do PIB do Agronegócio, quando se levam em consideração os valores dos insumos e não o valor adicionado efetivamente gerado na produção destes, foi eliminado. Tem-se então:

\footnotetext{
${ }^{6}$ O Valor Adicionado a preços de mercado é obtido pela soma do valor adicionado a preços básicos aos impostos indiretos líquidos de subsídios sobre produtos, resultando na seguinte expressão:

VAPM $=$ VAPB + IIL

Sendo: VAPM $=$ Valor Adicionado a Preços de Mercado

VAPB = Valor Adicionado a Preços Básicos

IIL = Impostos Indiretos Líquidos
} 


$$
P I B_{I_{k}}=\sum_{i=1}^{n} z_{i k} * C V A_{i}
$$

$k=1,2$ setor agricultura e pecuária

$i=1,2, \ldots$, n setores restantes

onde:

$P I B_{I_{k}}=$ PIB do agregado I (insumos) para agricultura $(k=1)$ e pecuária $(k=2)$

$z_{i k}=$ valor total do insumo do setor $i$ para a agricultura ou pecuária

$C V A_{i}=$ coeficiente de valor adicionado do setor $i$

Para o Agregado I total tem-se:

$P I B_{I}=P I B_{I_{1}}+P I B_{I_{2}}$

onde:

$P I B_{I}=$ PIB do agregado I e as outras variáveis são como definidas anteriormente.

Para o Agregado II (propriamente, o Setor da Agricultura e Pecuária) consideram-se no cálculo os valores adicionados gerados pelos respectivos setores e subtraem-se dos valores adicionados destes setores os valores que foram utilizados como insumos, eliminando-se o problema de dupla contagem presente em estimativas anteriores do PIB do Agronegócio. Tem-se então que:

$$
\begin{aligned}
& P I B_{I I_{k}}=V A_{P M_{k}}-\sum_{i=1}^{n} z_{i k} * C V A_{i} \\
& k=1,2
\end{aligned}
$$

onde:

$P I B_{I I_{k}}=$ PIB do agregado II para agricultura $k=1$, pecuária $k=2$ e as outras variáveis são como as definidas anteriormente. Para o Agregado II total tem-se:

$$
P I B_{I I}=P I B_{I I_{1}}+P I B_{I I_{2}}
$$

onde:

$P I B_{I I}=$ PIB do agregado IIM e as outras variáveis são como definidas anteriormente.

Para a definição da composição do Agregado III, as Indústrias de Base Agrícola, foram adotados vários indicadores, como por exemplo: a) os principais setores demandantes de produtos agrícolas, obtidos através da estimação da matriz de insumo-produto; b) as participações dos insumos agrícolas no consumo intermediário dos setores agroindustriais; e c) as atividades econômicas que 
efetuam a primeira, segunda e terceira transformações das matérias-primas agrícolas. Os Agregados II e III, portanto, expressam a renda ou o valor adicionado gerado por esses segmentos. No caso da estimação do Agregado III (Indústrias de Base Agrícola), adota-se o somatório dos valores adicionados pelos setores agroindustriais subtraídos dos valores adicionados destes setores que foram utilizados como insumos do Agregado II. Como mencionado, anteriormente, esta subtração visa eliminar a dupla contagem presente em estimativas anteriores do PIB do Agronegócio, ou seja:

$$
\begin{aligned}
& P I B_{I I I_{k}}=\sum_{q \in k}\left(V A_{P M_{q}}-z_{q k} * C V A_{q}\right) \\
& k=1,2
\end{aligned}
$$

onde:

$P I B_{I I I_{k}}=$ PIB do agregado III para agricultura $(k=1)$ e pecuária $(k=2)$ e as outras variáveis são como definidas anteriormente. Para o Agregado III total tem-se:

$$
P I B_{I I I}=P I B_{I I I_{1}}+P I B_{I I I_{2}}
$$

onde:

$P I B_{I I I}=$ PIB do agregado III e as outras variáveis são como as definidas anteriormente. Já, no caso do Agregado IV, referente à Distribuição Final, considerase para fins de cálculo o valor agregado dos setores relativos ao Transporte, Comércio e segmentos de Serviços. Do valor total obtido, destina-se ao Agronegócio apenas a parcela que corresponde à participação dos produtos agropecuários e agroindustriais na demanda final de produtos. A sistemática adotada no cálculo do valor da distribuição final do agronegócio industrial pode ser representada por:

$$
\begin{gathered}
D F G-I I L_{D F}-P I_{D F}=D F D \\
V A T_{P M}+V A C_{P M}+V A S_{P M}=M C \\
P I B_{I V_{k}}=M C^{*} \frac{D F_{k}+\sum_{q \in k} D F_{q}}{D F D} \\
k=1,2
\end{gathered}
$$

onde:

$D F G=$ demanda final global

IIL $L_{D F}=$ impostos indiretos líquidos pagos pela demanda final

$P I_{D F}=$ produtos importados pela demanda final

$D F D=$ demanda final doméstica

$V A T_{P M}=$ valor adicionado do setor transporte a preços de mercado 
$V A C_{P M}=$ valor adicionado do setor comércio a preços de mercado

$V A S_{P M}=$ valor adicionado do setor serviços a preços de mercado

$M C$ = margem de comercialização

$D F_{k}=$ demanda final da agricultura $(k=1)$ e pecuária $(k=2)$

$D F_{q}=$ demanda final dos setores agroindustriais

$P I B_{I V_{k}}=$ PIB do agregado IV para agricultura $(k=1)$ e pecuária $(k=2)$

Para o Agregado IV total tem-se:

$$
P I B_{I V}=P I B_{I V_{1}}+P I B_{I V_{2}}
$$

onde:

$P I B_{I V}=\mathrm{PIB}$ do agregado IV

O PIB total do Agronegócio é dado pela soma dos seus agregados, ou seja:

$$
\begin{aligned}
& P I B_{A g_{I_{k}}}=P I B_{I_{k}}+P I B_{I I_{k}}+P I B_{I I I_{k}}+P I B_{N_{V_{k}}} \\
& k=1,2
\end{aligned}
$$

onde:

$P I B_{A g r_{k}}=$ PIB do agronegócio para agricultura $(k=1)$ e pecuária $(k=2)$

Para o Agronegócio total tem-se:

$$
P I B_{A g r}=P I B_{A g r_{1}}+P I B_{A g r_{2}}
$$

onde:

$P I B_{A g r}=$ PIB do agronegócio

\section{Resultados e discussão}

Os resultados do Produto Interno Bruto do Agronegócio e sua participação no PIB estadual constam da Tabela 1. Os maiores valores absolutos são do estado de São Paulo com aproximadamente $\mathrm{R} \$ 146$ bilhões seguido por Minas Gerais e Rio Grande do Sul com aproximadamente $\mathrm{R} \$ 64$ bilhões cada um. Ainda de acordo com a Tabela 1 é possível observar a existência de grande variação da participação do Agronegócio nas economias estaduais, em valores percentuais da participação do Agronegócio no PIB estadual destacam-se Mato Grosso com 60\%, Mato Grosso do Sul com $42 \%$ e Rondônia com $40 \%$. Pela perspectiva inversa, os menores valores percentuais são do Distrito Federal (2\%), Rio de Janeiro (6\%), Amapá (9\%) e Roraima (12\%). O Mato Grosso apresenta a maior participação do Agronegócio em sua economia com $60 \%$, mas a participação da indústria no total do Agronegócio é baixa $(13 \%)$ e o estado poderia aumentar o processamento dos produtos básicos a 
fim de gerar valor adicionado. Esta análise vale para os estados de Acre, Rondônia, Maranhão, Tocantins e Mato Grosso do Sul. Por outro lado, Rio de Janeiro e São Paulo possuem produção agropecuária (Agregado II) relativamente pequena, $6 \% \mathrm{e}$ $8 \%$ respectivamente, em relação ao Agronegócio e são dependentes de produtos de outros estados, pois sua atividade industrial e alta em relação a participação dos demais estados, Rio de Janeiro com $49 \%$ e São Paulo $42 \%$. Os valores obtidos na pesquisa estão próximos aos verificados em estudos recentes para os estados do Brasil, respeitando-se o nível de agregação das atividades econômicas e período de análise. Deve-se considerar também que o método de construção da matriz e as fontes dos dados podem causar diferenças entre os resultados de outros trabalhos e este estudo. Porém, para a presente pesquisa as matrizes das unidades da federação utilizadas foram obtidas todas pelo mesmo método e isto torna possível a comparação de resultados entre as regiões para o ano de 2008.

A Figura 1 mostra o mapa dos estados com a frequência da participação do PIB do Agronegócio na economia de cada estado. Nota-se que os estados da Região Centro-oeste apresentaram maiores valores percentuais de participação do agronegócio na economia dentro da faixa de aproximadamente $36 \%$ a $60 \%$, seguida pela Região Sul com destaque para Santa Catarina.

A Figura 2 mostra a participação dos agregados no PIB do Agronegócio das unidades da federação. Nota-se as maiores variações da participação, com variância de 1,86\% e 1,2\% respectivamente, são da Agropecuária (Agregado II), que vai de $6 \%$ a $62 \%$, e da indústria (Agregado III), com valores que estão entre $5 \%$ a $49 \%$. No caso dos agregados I e IV, estes apresentaram variância próxima de zero, oscilando entre $1 \%$ a $7 \%$ de participação para os insumos para a agricultura e pecuária, e entre $27 \%$ a $48 \%$ no caso da distribuição final, considera-se para fins de cálculo o valor agregado dos setores relativos ao transporte, comércio e segmentos de serviços. Os resultados mostram que alguns estados poderiam aumentar a industrialização dos produtos agropecuários como Maranhão (5\%), Tocantins (9\%), Acre (10\%), Roraima (11\%), Rondônia (13\%), Mato Grosso (13\%) e Mato Grosso do Sul (17\%). Nestes estados a industrialização aumentaria a geração de valor adicionado e o valor do PIB do agronegócio de forma global em contraponto a comercialização dos bens com baixo processamento. A pesquisa mostra também que existem diferentes realidades para o Agronegócio entre os estados brasileiros, ou seja, quando observados os quatro agregados nenhum estado se destaca em 
mais de dois (sejam nas maiores ou menores participações) demonstrando grande variabilidade nas posições.

A Tabela 2 apresenta os resultados para a estimativa dos empregos existentes em cada agregado do Agronegócio nas economias dos estados e Distrito Federal. A participação da geração de empregos do Agronegócio no total dos estados está acima de 30\% para 21 unidades da federação, o que mostra a grande importância deste na maior parte das regiões analisadas. Porém, os valores indicam realidades diversas, pois enquanto em São Paulo o número de empregos na indústria e serviços é mais que o dobro do que na área rural, os estados do Mato Grosso, Acre e Amapá têm situação inversa com a maior parte dos empregos gerada no campo. Os valores para a geração de empregos no Agronegócio mostram que existe a possibilidade de aumentar o valor adicionado e postos de trabalho com aumento da industrialização e serviços dos produtos, como já apresentado anteriormente. 
Tabela 1. Produto Interno Bruto do Agronegócio em milhões de reais de 2008 e participação no Produto Interno Bruto estadual. Agregado I (Insumos), Agregado II (Agropecuária), Agregado III (Indústria) e Agregado IV (Serviços).

\begin{tabular}{|c|c|c|c|c|c|c|}
\hline \multirow{2}{*}{ Estados } & \multicolumn{4}{|c|}{ Agregados } & \multirow{2}{*}{ Total } & \multirow{2}{*}{$\begin{array}{c}\text { Participação } \\
(\%)\end{array}$} \\
\hline & I & II & III & IV & & \\
\hline \multicolumn{7}{|l|}{ Região Norte } \\
\hline Acre & 73 & 998 & 176 & 569 & 1.816 & 28 \\
\hline Amapá & 15 & 202 & 140 & 204 & 561 & 9 \\
\hline Amazonas & 77 & 1.696 & 3.169 & 1.825 & 6.766 & 16 \\
\hline Pará & 324 & 3.190 & 3.058 & 3.359 & 9.931 & 18 \\
\hline Rondônia & 231 & 3.063 & 888 & 2.454 & 6.636 & 40 \\
\hline Roraima & 35 & 259 & 64 & 205 & 562 & 12 \\
\hline Tocantins & 226 & 2.169 & 347 & 1.335 & 4.078 & 32 \\
\hline \multicolumn{7}{|l|}{ Região Nordeste } \\
\hline Alagoas & 172 & 1.202 & 1.105 & 1.823 & 4.302 & 23 \\
\hline Bahia & 1.331 & 7.283 & 4.964 & 9.402 & 22.981 & 20 \\
\hline Ceará & 416 & 3.266 & 5.112 & 7.895 & 16.690 & 29 \\
\hline Maranhão & 359 & 7.287 & 591 & 3.453 & 11.689 & 32 \\
\hline Paraíba & 114 & 1.278 & 1.721 & 2.668 & 5.780 & 24 \\
\hline Pernambuco & 407 & 2.880 & 3.563 & 5.915 & 12.764 & 19 \\
\hline Piauí & 175 & 1.439 & 757 & 1.569 & 3.940 & 25 \\
\hline Sergipe & 97 & 784 & 1.045 & 1.450 & 3.375 & 18 \\
\hline Rio Grande do & 118 & 943 & 1.459 & 2.157 & 4.676 & 19 \\
\hline \multicolumn{7}{|l|}{ Região Centro } \\
\hline Distrito Federal & 114 & 447 & 839 & 1.302 & 2.703 & 2 \\
\hline Goiás & 1.709 & 7.088 & 6.971 & 11.711 & 27.479 & 38 \\
\hline Mato Grosso & 2.056 & 12.308 & 4.066 & 11.717 & 30.147 & 60 \\
\hline Mato Grosso do Sul & 610 & 4.851 & 2.228 & 5.259 & 12.948 & 42 \\
\hline \multicolumn{7}{|l|}{ Região Sudeste } \\
\hline Espírito Santo & 300 & 3.504 & 2.498 & 4.626 & 10.927 & 18 \\
\hline Minas Gerais & 2.907 & 21.883 & 14.611 & 24.510 & 63.911 & 24 \\
\hline Rio de Janeiro & 250 & 1.162 & 8.983 & 7.938 & 18.333 & 6 \\
\hline São Paulo & 5.086 & 11.206 & 61.931 & 68.091 & 146.314 & 16 \\
\hline \multicolumn{7}{|l|}{ Região Sul } \\
\hline Paraná & 3.553 & 12.415 & 14.197 & 23.714 & 53.879 & 32 \\
\hline Santa Catarina & 1.676 & 8.022 & 14.096 & 17.771 & 41.566 & 36 \\
\hline Rio Grande do Sul & 2.913 & 16.461 & 16.097 & 28.514 & 63.985 & 34 \\
\hline
\end{tabular}

Fonte: cálculo dos autores. 
Figura 1. Frequência da participação do Produto Interno Bruto do Agronegócio na economia de cada estado em valores percentuais.

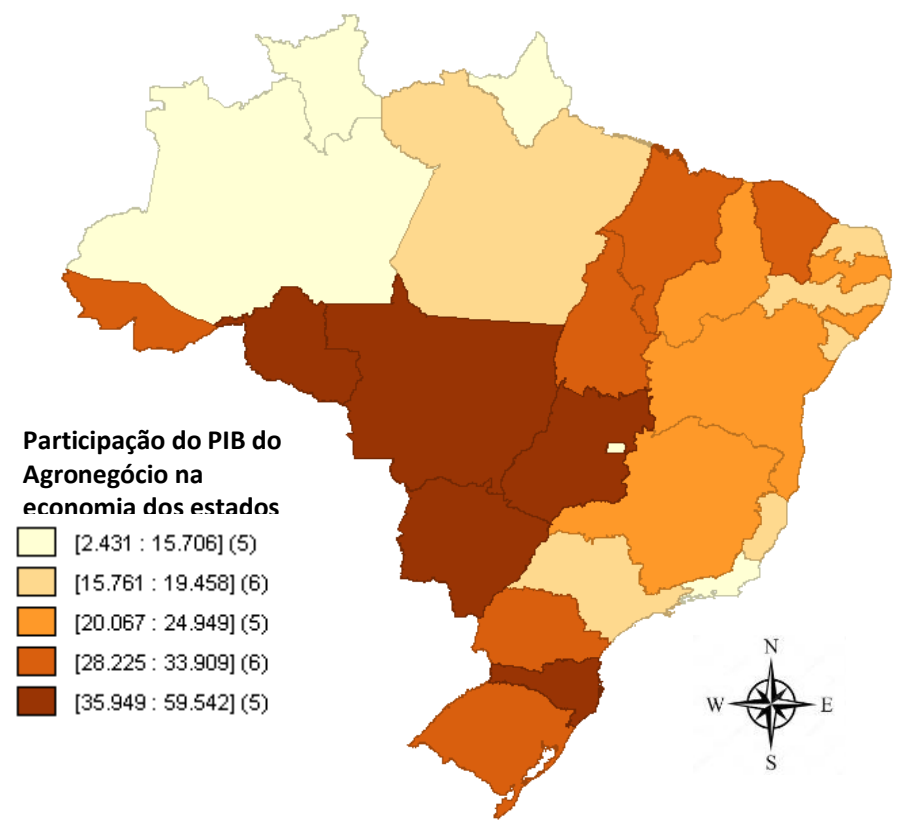

Fonte: elaborado pelos autores com resultados da pesquisa.

Figura 2. Participação (\%) dos agregados no Produto Interno Bruto do Agronegócio dos estados.

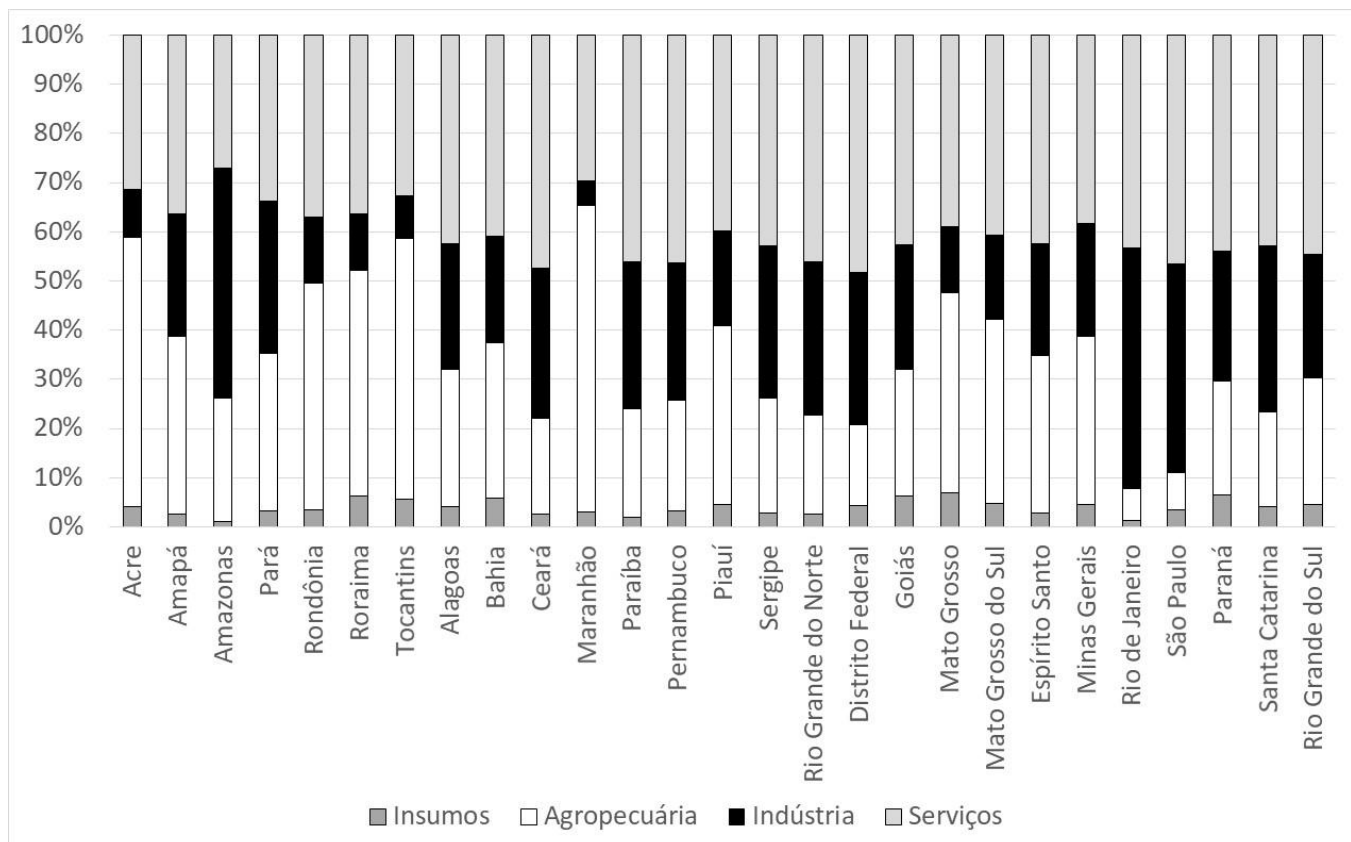

Fonte: elaborado pelos autores com resultados da pesquisa. 
Tabela 2. Geração de empregos do Agronegócio em 2008 e participação no total estadual. Agregado I (Insumos), Agregado II (Agropecuária), Agregado III (Indústria) e Agregado IV (Serviços).

\begin{tabular}{|c|c|c|c|c|c|c|}
\hline \multirow[b]{2}{*}{ Estados } & \multicolumn{4}{|c|}{ Agregados } & \multirow[b]{2}{*}{ Total } & \multirow{2}{*}{$\begin{array}{c}\text { Participaçã } \\
0 \\
(\%) \\
\end{array}$} \\
\hline & $\mathbf{I}$ & II & III & IV & & \\
\hline \multicolumn{7}{|l|}{ Região Norte } \\
\hline Acre & 5.504 & 90.971 & 10.349 & 24.580 & 131.403 & 40 \\
\hline Amapá & 960 & 20.446 & 6.543 & 8.011 & 35.960 & 13 \\
\hline Amazonas & 5.292 & 232.463 & 94.262 & 86.231 & 418.248 & 26 \\
\hline Pará & 29.477 & 478.209 & 172.751 & 168.574 & 849.011 & 32 \\
\hline Rondônia & 16.611 & 296.213 & 47.495 & 107.773 & 468.091 & 52 \\
\hline Roraima & 2.627 & 31.045 & 3.871 & 7.876 & 45.418 & 22 \\
\hline Tocantins & 18.019 & 264.985 & 20.080 & 68.728 & 371.812 & 49 \\
\hline \multicolumn{7}{|l|}{ Região Nordeste } \\
\hline Alagoas & 12.486 & 388.033 & 53.905 & 94.367 & 548.791 & 45 \\
\hline Bahia & 111.706 & 1.649 .296 & 277.268 & 465.379 & 2.503 .649 & 40 \\
\hline Ceará & 52.787 & 898.605 & 473.453 & 450.065 & 1.874 .909 & 47 \\
\hline Maranhão & 41.961 & 1.311 .682 & 39.320 & 211.060 & 1.604 .024 & 52 \\
\hline Paraíba & 12.998 & 315.262 & 115.899 & 144.264 & 588.422 & 38 \\
\hline Pernambuco & 32.744 & 799.710 & 176.749 & 283.605 & 1.292 .808 & 36 \\
\hline Piauí & 28.168 & 431.672 & 43.026 & 98.915 & 601.781 & 45 \\
\hline Sergipe & 10.757 & 191.670 & 65.559 & 72.877 & 340.863 & 36 \\
\hline Rio Grande do Norte & 11.704 & 223.019 & 121.320 & 118.831 & 474.873 & 34 \\
\hline \multicolumn{7}{|l|}{ Região Centro Oeste } \\
\hline Distrito Federal & 3.953 & 36.088 & 22.436 & 26.831 & 89.308 & 4 \\
\hline Goiás & 85.388 & 754.315 & 221.299 & 434.301 & 1.495 .303 & 50 \\
\hline Mato Grosso & 92.147 & 969.733 & 124.684 & 386.758 & 1.573 .322 & 70 \\
\hline Mato Grosso do Sul & 34.423 & 366.397 & 88.398 & 200.155 & 689.373 & 51 \\
\hline \multicolumn{7}{|l|}{ Região Sudeste } \\
\hline Espírito Santo & 10.189 & 208.063 & 79.061 & 142.624 & 439.937 & 27 \\
\hline Minas Gerais & 117.950 & 1.359 .871 & 642.507 & 872.244 & 2.992 .572 & 34 \\
\hline Rio de Janeiro & 7.720 & 84.125 & 292.723 & 214.364 & 598.932 & 8 \\
\hline São Paulo & 117.537 & 941.111 & 2.244 .031 & 1.736 .440 & 5.039 .119 & 22 \\
\hline \multicolumn{7}{|l|}{ Região Sul } \\
\hline Paraná & 141.744 & 1.167 .461 & 446.283 & 798.053 & 2.553 .542 & 42 \\
\hline Santa Catarina & 67.884 & 747.941 & 509.001 & 536.801 & 1.861 .627 & 49 \\
\hline Rio Grande do Sul & 129.496 & 1.658 .332 & 565.873 & 831.400 & 3.185 .102 & 49 \\
\hline
\end{tabular}

Fonte: cálculo dos autores.

Outra questão importante diz respeito a produtividade do trabalho, a Tabela 3 apresenta a produtividade do trabalho dos agregados do Agronegócio calculada como PIB por emprego. Os menores valores foram encontrados para os estados do Norte e Nordeste do Brasil e os maiores valores obtidos para os estados da Região 
Sudeste e para o Distrito Federal (na Região Centro Oeste). Em relação a este último, observa-se que cada unidade de mão de obra produz $\mathrm{R} \$ 48,50$ mil de PIB no agregado IV (transporte, comércio e segmentos de serviços).

Observando a Tabela 3, de forma geral os maiores valores de produtividade do trabalho foram obtidos para os agregados III (indústria) e IV (serviços), com destaque, além do Distrito Federal, para São Paulo ( $R \$ 39,20$ mil de PIB por trabalhador) e Rio de Janeiro com $\mathrm{R} \$ 37,00$ mil. Esses resultados indicam a importância destas atividades para o agronegócio. Ainda em relação a São Paulo é importante destacar o agregado I (insumos) com $R \$ 43,30$ mil, o segundo maior valor de produtividade dentre todos os agregados. No tocante a esse agregado é importante mencionar que este representa os setores de combustíveis, fertilizantes, defensivos, alimentos para animais, sal mineral e medicamentos veterinários, máquinas e equipamentos agrícolas. Além do que, mesmo representando um alto valor de produtividade, esse agregado para São Paulo é pouco mais de 3,4\% do PIB do Agronegócio do estado.

É importante observar que existe a possibilidade de aumentar a produtividade do trabalho com tecnologia, capital e qualificação profissional, notadamente em regiões que apresentaram valores próximos ou abaixo da média nacional $(R \$ 14,20$ mil de PIB por trabalhador), a exemplo dos estados do Norte e Nordeste, mas que apresentam uma trajetória de crescimento no agronegócio de forma global.

Tabela 3. Produtividade do trabalho medida em Produto Interno Bruto por emprego nos agregados do Agronegócio em 2008 em milhares de reais. Agregado I (Insumos), Agregado II (Agropecuária), Agregado III (Indústria) e Agregado IV (Serviços).

\begin{tabular}{|c|c|c|c|c|c|}
\hline \multirow{2}{*}{ Estados } & \multicolumn{4}{|c|}{ Agregados } & \multirow{2}{*}{ Total } \\
\hline & I & II & III & IV & \\
\hline \multicolumn{6}{|l|}{ Região Norte } \\
\hline Acre & 13,4 & 11,0 & 17,0 & 23,1 & 13,8 \\
\hline Amapá & 15,3 & 9,9 & 21,4 & 25,5 & 15,6 \\
\hline Amazonas & 14,5 & 7,3 & 33,6 & 21,2 & 16,2 \\
\hline Pará & 11,0 & 6,7 & 17,7 & 19,9 & 11,7 \\
\hline Rondônia & 13,9 & 10,3 & 18,7 & 22,8 & 14,2 \\
\hline Roraima & 13,2 & 8,3 & 16,4 & 26,0 & 12,4 \\
\hline Tocantins & 12,5 & 8,2 & 17,3 & 19,4 & 11,0 \\
\hline \multicolumn{6}{|c|}{ Região Nordeste } \\
\hline Alagoas & 13,8 & 3,1 & 20,5 & 19,3 & 7,8 \\
\hline Bahia & 11,9 & 4,4 & 17,9 & 20,2 & 9,2 \\
\hline Ceará & 7,9 & 3,6 & 10,8 & 17,5 & 8,9 \\
\hline Maranhão & 8,5 & 5,6 & 15,0 & 16,4 & 7,3 \\
\hline Paraíba & 8,8 & 4,1 & 14,8 & 18,5 & 9,8 \\
\hline Pernambuco & 12,4 & 3,6 & 20,2 & 20,9 & 9,9 \\
\hline
\end{tabular}




\begin{tabular}{|c|c|c|c|c|c|}
\hline Piauí & 6,2 & 3,3 & 17,6 & 15,9 & 6,5 \\
\hline Sergipe & 9,0 & 4,1 & 15,9 & 19,9 & 9,9 \\
\hline Rio Grande do Norte & 10,0 & 4,2 & 12,0 & 18,2 & 9,8 \\
\hline \multicolumn{6}{|l|}{ Região Centro } \\
\hline Distrito Federal & 28,9 & 12,4 & 37,4 & 48,5 & 30,3 \\
\hline Goiás & 20,0 & 9,4 & 31,5 & 27,0 & 18,4 \\
\hline Mato Grosso & 22,3 & 12,7 & 32,6 & 30,3 & 19,2 \\
\hline Mato Grosso do Sul & 17,7 & 13,2 & 25,2 & 26,3 & 18,8 \\
\hline \multicolumn{6}{|l|}{ Região Sudeste } \\
\hline Espírito Santo & 29,4 & 16,8 & 31,6 & 32,4 & 24,8 \\
\hline Minas Gerais & 24,6 & 16,1 & 22,7 & 28,1 & 21,4 \\
\hline Rio de Janeiro & 32,4 & 13,8 & 30,7 & 37,0 & 30,6 \\
\hline São Paulo & 43,3 & 11,9 & 27,6 & 39,2 & 29,0 \\
\hline \multicolumn{6}{|l|}{ Região Sul } \\
\hline Paraná & 25,1 & 10,6 & 31,8 & 29,7 & 21,1 \\
\hline Santa Catarina & 24,7 & 10,7 & 27,7 & 33,1 & 22,3 \\
\hline Rio Grande do Sul & 22,5 & 9,9 & 28,4 & 34,3 & 20,1 \\
\hline
\end{tabular}

Fonte: cálculo dos autores.

Em resumo, os resultados das Tabelas 1, 2 e 3 indicam que o aumento da complexidade do agronegócio com a industrialização e serviços adicionados ao processo de comercialização permitem alcançar maior geração de renda e de empregos. Cadeias produtivas que possuem na mesma região as atividades de fornecimento de insumos, produção agropecuária, industrialização e serviços são capazes de fornecer postos de trabalho com maior geração de valor adicionado. Contudo, os dados indicam uma disparidade no tocante a eficiência técnica entre os estados, o que demandaria uma estratégia de política pública de forma a mitigar essa realidade.

A Tabela 4 apresenta os resultados para a geração de impostos indiretos líquidos do Agronegócio. Observa-se a importância em termos de participação no valor dos impostos totais, os maiores percentuais são para Mato Grosso, Mato Grosso do Sul, Goiás, Paraná, Santa Catarina, Rio Grande do Sul, Rondônia e Tocantins. Para estes estados a participação do Agronegócio na geração de impostos indiretos líquidos totais varia entre $35 \%$ e $66 \%$. O caso do Mato Grosso é muito específico, $66 \%$ da receita líquida é obtida dentro da cadeia produtiva do agronegócio.

É importante lembrar que as commodities agrícolas apresentam alta variação dos preços, os quais normalmente são formados nos mercados internacionais. 
Nesse sentido dois pontos devem ser observados. Primeiro, os preços voláteis das commodities agropecuárias podem tornar a receita líquida estadual muito variável. Portanto, anos em que os preços internacionais caem os estados altamente dependentes desta receita terão problemas em equilibrar as contas. Outra questão importante é a participação dos impostos no total do Produto Interno Bruto em cada agregado do Agronegócio, os valores foram calculados e os resultados foram utilizados para elaborar a Tabela 5 .

Ainda em relação a Tabela 5, esta mostra os valores percentuais de participação dos impostos em relação ao PIB do Agronegócio em cada estado desagregado por elos da cadeia produtiva. Os valores de participação variam entre $3 \%$ a mais de $30 \%$. Isto mostra que os estados tratam de forma muito diferente a tributação para as diversas atividades do Agronegócio. A unidade da federação com maiores percentuais de impostos no PIB do Agronegócio é o Distrito Federal com $18 \%$, seguido por São Paulo e Rio de Janeiro com 13\% e Mato Grosso do Sul e Espírito Santo com $12 \%$. Os resultados mostram que existiria possibilidade de desonerar o sistema produtivo para estes estados. Observando o Agregado I (insumos), este elo da cadeia produtiva mostra maior tributação nos estados de Distrito Federal, Rio de Janeiro, São Paulo e Pernambuco. Para a produção agropecuária, a maior tributação ocorre em Distrito Federal e São Paulo. $O$ agregado III (indústria) sofre a maior parte da tributação em valores percentuais no PIB do Agronegócio variando entre 9\% e 33\%, maiores valores para Distrito Federal, Mato Grosso do Sul e Espírito Santo. O quarto agregado (comércio e serviços) possui os menores valores variando entre $3 \%$ e $8 \%$ com maiores percentuais para Mato Grosso do Sul e Espírito Santo.

Respeitando o fato de que os produtos do Agronegócio são diferentes entre as unidades da federação, ainda é possível afirmar que existe a possibilidade de analisar os maiores valores encontrados na pesquisa para a desoneração dos produtos do Agronegócio principalmente bens industrializados. Considerando que os valores tributados serão incorporados no preço final dos produtos, a desoneração traria benefícios para a população com menores preços e maior competitividade ao Agronegócio, além de beneficiar a população de baixa renda que compromete parte considerável da renda com alimentação. 
Tabela 4. Impostos Indiretos Líquidos do Agronegócio em milhões de reais de 2008 e participação no total estadual. Agregado I (Insumos), Agregado II (Agropecuária), Agregado III (Indústria) e Agregado IV (Serviços).

\begin{tabular}{|c|c|c|c|c|c|c|}
\hline \multirow[t]{2}{*}{ Estados } & \multicolumn{4}{|c|}{ Agregados } & \multirow[t]{2}{*}{ Total } & \multirow[t]{2}{*}{$\begin{array}{c}\text { Participação } \\
(\%)\end{array}$} \\
\hline & 1 & II & III & IV & & \\
\hline \multicolumn{7}{|l|}{ Região Norte } \\
\hline Acre & 3 & 26 & 24 & 16 & 69 & 29 \\
\hline Amapá & 1 & 6 & 12 & 5 & 24 & 11 \\
\hline Amazonas & 7 & 61 & 494 & 98 & 660 & 13 \\
\hline Pará & 22 & 188 & 550 & 146 & 906 & 24 \\
\hline Rondônia & 10 & 83 & 142 & 70 & 305 & 41 \\
\hline Roraima & 2 & 14 & 9 & 7 & 31 & 16 \\
\hline Tocantins & 11 & 116 & 75 & 59 & 262 & 37 \\
\hline \multicolumn{7}{|l|}{ Região Nordeste } \\
\hline Alagoas & 18 & 46 & 182 & 85 & 331 & 29 \\
\hline Bahia & 164 & 220 & 860 & 437 & 1.682 & 18 \\
\hline Ceará & 36 & 129 & 852 & 415 & 1.433 & 35 \\
\hline Maranhão & 22 & 189 & 134 & 189 & 534 & 23 \\
\hline Paraíba & 8 & 50 & 266 & 115 & 438 & 30 \\
\hline Pernambuco & 54 & 138 & 760 & 369 & 1.321 & 23 \\
\hline Piauí & 10 & 57 & 137 & 64 & 269 & 32 \\
\hline Sergipe & 8 & 28 & 133 & 63 & 232 & 21 \\
\hline Rio Grande do & 12 & 54 & 184 & 117 & 366 & 22 \\
\hline \multicolumn{7}{|l|}{ Região Centro } \\
\hline Distrito Federal & 20 & 90 & 280 & 83 & 474 & 6 \\
\hline Goiás & 172 & 579 & 1.721 & 556 & 3.027 & 48 \\
\hline Mato Grosso & 141 & 607 & 1.069 & 470 & 2.287 & 66 \\
\hline Mato Grosso do Sul & 51 & 452 & 689 & 323 & 1.515 & 51 \\
\hline \multicolumn{7}{|l|}{ Região Sudeste } \\
\hline Espírito Santo & 40 & 197 & 681 & 366 & 1.284 & 19 \\
\hline Minas Gerais & 284 & 761 & 2.747 & 1.218 & 5.010 & 23 \\
\hline Rio de Janeiro & 44 & 73 & 1.650 & 631 & 2.398 & 7 \\
\hline São Paulo & 811 & 1.145 & 12.806 & 4.728 & 19.490 & 19 \\
\hline \multicolumn{7}{|l|}{ Região Sul } \\
\hline Paraná & 447 & 770 & 2.830 & 1.148 & 5.196 & 35 \\
\hline Santa Catarina & 140 & 644 & 2.214 & 760 & 3.758 & 43 \\
\hline Rio Grande do Sul & 309 & 709 & 3.445 & 1.462 & 5.925 & 35 \\
\hline
\end{tabular}

Fonte: cálculo dos autores.

Tabela 5. Participação dos Impostos Indiretos Líquidos no PIB Agronegócio (valores em \%). Agregado I (Insumos), Agregado II (Agropecuária), Agregado III (Indústria) e Agregado IV (Serviços).

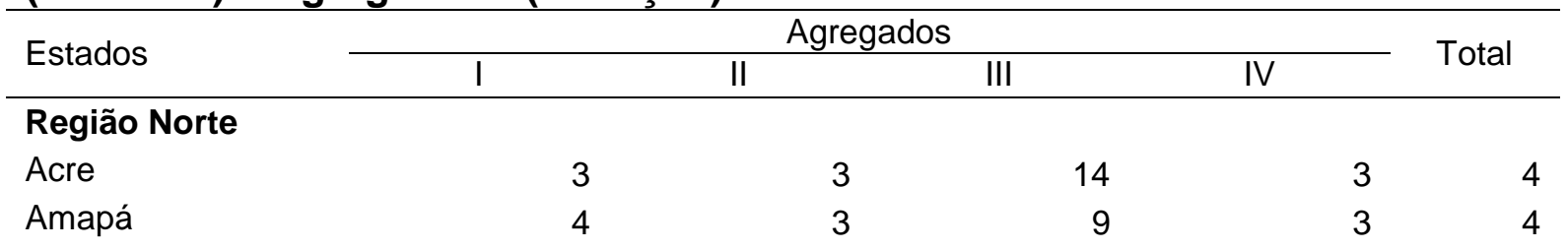


Amazonas

Pará

Rondônia

Roraima

Tocantins

\section{Região Nordeste}

Alagoas

Bahia

Ceará

Maranhão

Paraíba

Pernambuco

Piauí

Sergipe

Rio Grande do

Norte

\section{Região Centro}

Distrito Federal

Goiás

Mato Grosso

Mato Grosso do Sul

Região Sudeste

Espírito Santo

Minas Gerais

Rio de Janeiro

São Paulo

Região Sul

Paraná

Santa Catarina

Rio Grande do Sul

Fonte: cálculo dos autores.

$\begin{array}{rrrrr}9 & 4 & 16 & 5 & 10 \\ 7 & 6 & 18 & 4 & 9 \\ 4 & 3 & 16 & 3 & 5 \\ 5 & 5 & 14 & 3 & 6 \\ 5 & 5 & 22 & 4 & 6\end{array}$

$\begin{array}{rrrrr}11 & 4 & 16 & 5 & 8 \\ 12 & 3 & 17 & 5 & 7 \\ 9 & 4 & 17 & 5 & 9 \\ 6 & 3 & 23 & 5 & 5 \\ 7 & 4 & 15 & 4 & 8 \\ 13 & 5 & 21 & 6 & 10 \\ 6 & 4 & 18 & 4 & 7 \\ 8 & 4 & 13 & 4 & 7 \\ 10 & 6 & 13 & 5 & 8\end{array}$

$\begin{array}{rrrrr}18 & 20 & 33 & 6 & 18 \\ 10 & 8 & 25 & 5 & 11 \\ 7 & 5 & 26 & 4 & 8 \\ 8 & 9 & 31 & 6 & 12\end{array}$

$\begin{array}{lll}13 & 6 & 27\end{array}$

12

$\begin{array}{lll}10 & 3 & 19\end{array}$

$\begin{array}{lll}18 & 6 & 18\end{array}$

8

13

$7 \quad 13$

16

10

21

\section{REFERÊNCIAS}

ARAUJO NETO, D.L.; COSTA, E.F. Dimensionamento do PIB do agronegócio em Pernambuco, Rev. Econ. Sociol. Rural, Brasília, v. 43, n. 4, p. 725-757, Dec. 2005. Disponível em <http://www.scielo.br/scielo.php?script=sci_arttext\&pid=S0103$20032005000400006 \&$ Ing=en\&nrm=iso $>$. Acesso em 28 de agosto de 2017. http://dx.doi.org/10.1590/S0103-20032005000400006.

CEPEA. Centro de Estudos Avançados em Economia Aplicada (CEPEA/ESALQ/USP). http://www.cepea.esalq.usp.br/. Acessado em 29/08/2017.

CRUZ, A.C.; TEIXEIRA, E.C.; GOMES, M.F.M. O PIB do agronegócio no estado de Minas Gerais: uma análise insumo-produto, Revista de Economia e Sociologia Rural, Brasília, v.47, n.4, p.805-830, Dec.2009. Disponível em $<$ http://www.scielo.br/scielo.php?script=sci_arttext\&pid=S0103- 
$20032009000400001 \& \operatorname{lng}=$ en\&nrm=iso $>$. Acesso em 28 de agosto de 2017. http://dx.doi.org/10.1590/S0103-20032009000400001.

DAVIS, J.H.; GOLDBERG, R. A Concept of Agribusiness. Boston, Harvard University, 1957.

FINAMORE, E. B., MONTOYA, M. A. PIB, Tributos, Emprego, Salários e Saldo Comercial no Agronegócio Gaúcho. Ensaios FEE, Porto Alegre, v. 24, n. 1, p. 93-126, 2003.

FURTUOSO, M. O Produto Interno Bruto do Complexo Agroindustrial Brasileiro. Tese (Doutorado) - Esalq/USP, 1998.

FURTUOSO, M.C.O.; GUILHOTO, J.J.M. Estimativa e Mensuração do Produto Interno Bruto do Agronegócio da Economia Brasileira 1994 a 2000. Revista Brasileira de Economia e Sociologia Rural, v. 43, n.4, 2003.

GUILHOTO, J. J. M.; ASSUMPÇÃO, M.; MODOLO, D.; IMORI, D. O PIB do Agronegócio no Brasil e no Estado da Bahia. Anais do XLV Congresso da Sociedade Brasileira de Economia, Administração e Sociologia Rural, Londrina, 2007.

GUILHOTO, J.J.M. E U. A. SESSO FILHO. Estimação da Matriz Insumo-Produto a Partir de Dados Preliminares das Contas Nacionais. Economia Aplicada. Vol. 9. N. 2. Abril-Junho. pp. 277-299, 2005.

GUILHOTO, J.J.M., C.R. AZZONI, S.M. ICHIHARA, D.K. KADOTA, E.A. HADDAD. Matriz de Insumo-Produto do Nordeste e Estados: Metodologia e Resultados. Fortaleza: Banco do Nordeste do Brasil. ISBN: 978.85.7791.110.3. 289 p. 2010.

GUILHOTO, J.J.M., U.A. SESSO FILHO. Estimação da Matriz Insumo-Produto Utilizando Dados Preliminares das Contas Nacionais: Aplicação e Análise de Indicadores Econômicos para o Brasil em 2005. Economia \& Tecnologia. UFPR/TECPAR. Ano 6, Vol 23, Out./Dez., 2010. ISSN 1809-080X.

GUILHOTO, J.J.M.; AZZONI, C.R.; SILVEIRA, F.G., ICHIHARA, S.M.; Diniz, B. P. C.; Moreira, G.R.C. PIB Da Agricultura Familiar: Brasil - Estados. Disponível em: https://ssrn.com/abstract=1803225. Acesso em 15 dez. 2018. http://dx.doi.org/10.2139/ssrn.1803225

MORETTO, A. C., RODRIGUES, R. L., SESSO FILHO, U. A., MAIA, K. O Paraná na dinâmica da renda do sistema inter-regional Sul-Restante do Brasil. Economia \& tecnologia (UFPR)., v.15, p.65 - 75, 2008.

NEREUS. Núcleo de Economia Regional da Universidade de São Paulo. Disponível em: http://www.usp.br/nereus/. Acesso em 22/08/2017.

NUNES, P. A.; PARRÉ, J.L. Dimensionamento do agronegócio paranaense: 2007. Faz Ciência (UNIOESTE. Impresso), v. 15, p. 126-142, 2013.

PORSSE, A.A. Matriz de insumo-produto do Rio Grande do Sul - 2003. Porto Alegre: Fundação de Economia e Estatística Siegfried Emanuel Heuser, 2007. v. 1. 216 p.

PORSSE, A.A. Notas metodológicas sobre o dimensionamento do PIB do agronegócio do Rio Grande do Sul. Porto Alegre: Fundação de Economia e Estatística Siegfried Emanuel Heuser, 2003. 33p.

SESSO FILHO, U.A.; GUILHOTO, J.J.M.; RODRIGUES, R.L.; MORETTO, A.C.; GOMES, M.R. Geração de renda, emprego e impostos no agronegócio dos estados da Região Sul e restante do Brasil. Economia \& tecnologia (UFPR), v. 25, p. 71-80, 2011. 


\section{NOTAS DE AUTOR}

\section{CONTRIBUIÇÃO DE AUTORIA}

Umberto Antonio Sesso Filho e Lucas Trindade Borges - Concepção. Coleta de dados, Análise de dados, Elaboração do manuscrito, revisão e aprovação da versão final do trabalho

Patrícia Pompermayer Sesso, Irene Domenes Zapparoli e Paulo Rogério Alves Brene -Participação ativa da discussão dos resultados; Revisão e aprovação da versão final do trabalho.

\section{FINANCIAMENTO}

Lucas Trindade Borges é aluno do curso de Mestrado em Economia Regional com bolsa da Fundação AraucáriaCAPES. O Projeto de pesquisa desenvolvido no Departamento de Economia da Universidade Estadual de Londrina recebeu apoio financeiro da Fundação Araucária e CNPq.

\section{CONSENTIMENTO DE USO DE IMAGEM}

Não se aplica.

APROVAÇÃO DE COMITÊ DE ÉTICA EM PESQUISA

Não se aplica.

\section{CONFLITO DE INTERESSES}

Não se aplica.

\section{LICENÇA DE USO}

Este artigo está licenciado sob a Licença Creative Commons CC-BY. Com essa licença você pode compartilhar, adaptar, criar para qualquer fim, desde que atribua a autoria da obra.

\section{HISTÓRICO}

Recebido em: 30-09-2018

Aprovado em: 07-02-2019 